\title{
Full Elasticity Tensor from Thermal Diffuse Scattering
}

\author{
Björn Wehinger, ${ }^{1,2, *}$ Alessandro Mirone, ${ }^{3, \dagger}$ Michael Krisch, ${ }^{3}$ and Alexeï Bosak ${ }^{3}$ \\ ${ }^{1}$ Department of Quantum Matter Physics, University of Geneva, 24, Quai Ernest Ansermet, CH-1211 Genève, Switzerland \\ ${ }^{2}$ Laboratory for Neutron Scattering and Imaging, Paul Scherrer Institute, CH-5232 Villigen PSI, Switzerland \\ ${ }^{3}$ European Synchrotron Radiation Facility, 71, Avenue des Martyrs, F-38000 Grenoble, France
}

(Received 30 August 2016; published 20 January 2017)

\begin{abstract}
We present a method for the precise determination of the full elasticity tensor from a single crystal diffraction experiment using monochromatic $\mathrm{x}$ rays. For the two benchmark systems calcite and magnesium oxide, we show that the measurement of thermal diffuse scattering in the proximity of Bragg reflections provides accurate values of the complete set of elastic constants. This approach allows for a reliable and model-free determination of the elastic properties and can be performed together with crystal structure investigation in the same experiment.
\end{abstract}

DOI: 10.1103/PhysRevLett.118.035502

The elasticity tensor is the fundamental quantity for describing elastic waves and determines eventual anisotropic compression and sound velocities in crystalline materials [1]. The elastic properties define mechanical properties of materials and influence phase stability [2]. Accurate measurements of the elasticity tensor are of principal interest for the description of seismological waves and their discontinuities, which allow for decisive conclusions on composition, temperature, and pressure of Earth's interior [3]. In condensed matter physics elastic properties are important in the study of quantum phase transitions in systems with pronounced interaction of phonons with other quasiparticles. Such interaction may include electron-phonon coupling with applicability to superconductors [4-6] as well as spin-phonon coupling with interesting anomalies in lowdimensional spin systems $[7,8]$. The most commonly used experimental techniques to determine the coefficients of the elasticity tensor - the so-called elastic constants-are ultrasound measurements and Brillouin scattering. Ultrasound measurements are limited to relatively large crystals with well-defined faces; measurements at extreme conditions such as high pressures or high magnetic fields are very challenging. Brillouin scattering can be performed at high pressures but is difficult for opaque materials. Alternatively, the elasticity tensor can be extracted from inelastic x-ray or neutron scattering, which becomes efficient for crystals of low symmetry if combined with calculations from first principles [9]. Inelastic x-ray scattering can be performed at extreme pressures using diamond anvil cells and can be combined with low or high temperatures [10,11].

Ratios of elastic constants can also be obtained from thermal diffuse scattering (TDS) measured with energy integrating detectors, and a complete formalism was derived in the 1960s [12]. Historically, the thermal nature of diffuse scattering was noticed already in the 1920s [13,14], and the first phonon dispersion relations were in fact determined from TDS of $x$ rays [15]. The currently available high-flux and brilliant $\mathrm{x}$-ray beams from synchrotrons in combination with bidimensional single photon counting $\mathrm{x}$-ray detectors with good quantum efficiency and no readout noise has attracted new interest in TDS studies [16-18]. By the use of force constant models it is possible to determine the phonon dispersion relations, while a model-free reconstruction of the lattice dynamics can be realized only for monoatomic crystals [19].

In this Letter, we present a method for the precise determination of the full elasticity tensor from a single crystal diffraction experiment using a model-free data analysis for arbitrary crystal symmetries. For the two benchmark systems calcite (trigonal) and magnesium oxide (cubic), we show that the measurement of thermal diffuse scattering at two close temperatures is sufficient to obtain the full elasticity tensor in absolute units within remarkable accuracy. We discuss the influence of additional contributions to the diffuse scattering and evaluate multiple phonon scattering with the help of first-principles calculations using density functional perturbation theory.

Diffuse x-ray scattering intensities were collected in transmission geometry using a single photon counting pixel detector with no readout noise and large dynamical range. High-quality single crystals were prepared by mechanical cutting and polishing; surface defects were removed by gentle etching. Temperature was controlled by a nitrogen cryostream. The experiment on calcite was conducted on ID29 at the European Synchrotron Radiation Facility (ESRF) [20]. The sample was a rectangular bar with dimensions of approximately $50 \mu \mathrm{m} \times 50 \mu \mathrm{m} \times 1.5 \mathrm{~mm}$. Measurements were taken at $170 \mathrm{~K}$. Monocromatic $\mathrm{x}$ rays with a wavelength of $\lambda=0.6968 \AA$ and a microfocus of $60 \times 30 \mu \mathrm{m}^{2}$ were used. The sample was rotated from 0 to $100^{\circ}$ orthogonal to the beam with angular steps of $0.1^{\circ}$. The exposure time was $1.0 \mathrm{sec}$ per frame at a flux of $2.4 \times 10^{11}$ photons per sec (total collection time $17 \mathrm{~min}$ ). Scattering intensities were collected with a PILATUS 6M detector (Dectris, Baden, Switzerland), equipped with $300 \mu \mathrm{m}$ thick Si pixels of size $172^{2} \mu \mathrm{m}^{2}$, at a sample-detector distance of $300 \mathrm{~mm}$. Measurements on 
magnesium oxide were performed at the Swiss Norwegian beam line BM01A at the ESRF, where we used a cubic single crystal with edge length of $2 \mathrm{~mm}$. The sample was measured at $\lambda=0.68894 \AA$ and temperatures $90 \mathrm{~K}$ and $120 \mathrm{~K}$. The crystal was rotated by $360^{\circ}$ with the same angular step as for calcite. Scattering intensities were recorded with a PILATUS $2 \mathrm{M}$ detector at a distance of $244 \mathrm{~mm}$ from the sample. The exposure was $0.4 \mathrm{sec}$ per frame at a flux of $3.0 \times 10^{10}$ photons per sec (total collection time $48 \mathrm{~min}$ ).

Assuming the validity of both adiabatic and harmonic approximations, the intensity of $\mathrm{x}$-ray scattering from phonons for single- and two-phonon processes is given by

$$
I_{1}(\boldsymbol{Q})=\frac{\hbar N I_{\mathrm{inc}}}{2} \sum_{\nu} \Omega_{\boldsymbol{q}, \nu}\left|\sum_{s} \frac{f_{s}(\boldsymbol{Q})}{\sqrt{m_{s}}} e^{-W_{s, Q}}\left(\boldsymbol{Q} \boldsymbol{e}_{\boldsymbol{Q}, \nu, s}\right) e^{-i \boldsymbol{Q} \boldsymbol{\tau}_{s}}\right|^{2}
$$

and

$$
\begin{aligned}
I_{2}(\boldsymbol{Q})= & \frac{\hbar^{2} N V I_{\mathrm{inc}}}{8} \int \frac{d^{3} \boldsymbol{q}}{(2 \pi)^{3}} \sum_{\nu, \nu^{\prime}} \Omega_{\boldsymbol{q}, \nu} \Omega_{\boldsymbol{Q}-\boldsymbol{q}, \nu^{\prime}} \\
& \times\left|\sum_{s} \frac{f_{s}(\boldsymbol{Q})}{m_{s}} e^{-W_{s, \boldsymbol{Q}}} e^{-i \boldsymbol{Q} \boldsymbol{\tau}_{s}}\left(\boldsymbol{Q} \boldsymbol{e}_{\boldsymbol{q}, \nu, s}\right)\left(\boldsymbol{Q} \boldsymbol{e}_{\boldsymbol{Q}-\boldsymbol{q}, \nu, s}\right)\right|^{2},
\end{aligned}
$$

with

$$
\Omega_{q, \nu}=\frac{1}{\omega_{q, \nu}} \operatorname{coth}\left(\frac{\hbar \omega_{q, \nu}}{2 k_{B} T}\right),
$$

respectively; see Refs. [17,18] for a description in modern notation. Here, $N$ is the number of unit cells, $I_{\text {inc }}$ the incident beam intensity, and $f$ the atomic scattering factor of ion $s$ with mass $m$ and (anisotropic) Debye-Waller factor $W . \omega$ denotes the eigenfrequency and $\boldsymbol{e}$ the eigenvector of the phonon at reduced momentum transfer $\boldsymbol{q}=\boldsymbol{Q}-\boldsymbol{\tau}$ and branch $\nu . \boldsymbol{Q}$ is the total scattering vector, $\boldsymbol{\tau}$ the atomic basis vector within the unit cell, $V$ the unit cell volume, $T$ the temperature, and $k_{B}$ the Boltzmann constant. For small $q$ the single phonon scattering is dominated by the contribution of acoustic phonons with $I_{1}(\boldsymbol{Q}) \sim 1 / \omega_{\boldsymbol{q}, \nu}^{2}$.

Within the theory of elastic waves in crystals, the equation of motion is given by

$$
\rho \omega^{2} u_{i}=c_{i j l m} k_{j} k_{l} u_{m},
$$

where $c_{i j l m}$ is the tensor of elastic constants, $\rho$ the mass density, and $\boldsymbol{k}=\boldsymbol{k} \boldsymbol{n}$ and $\omega$ are the wave vector and the frequency of the elastic waves, respectively [1].

For fitting the elastic constants to the experimental intensities in the vicinity of Bragg reflections, we calculate the scattering intensities as the sum of the contributions from the three acoustic branches. We therefore solve the equation of motion [Eq. (4)] for a given crystal symmetry and calculate the scattering intensities by summing over the three acoustic phonon branches in Eqs. (1) and (2). The thus-obtained intensities are renormalized by an array $\boldsymbol{g}(\boldsymbol{Q})$ that accounts for absorption, polarization, and geometrical corrections. An array $\boldsymbol{b}(\boldsymbol{Q})$ is added for the background.

Given a set of experimental intensities $I_{Q . T}^{\text {expt }}$ measured at $T$ over a set of reciprocal space points $\boldsymbol{Q} \in\left\{\boldsymbol{Q}_{\text {expt }}\right\}$ in the proximity to Bragg reflections, we find the elasticity tensor $c$ by solving the optimization problem,

$$
c, \boldsymbol{b}, \boldsymbol{g}=\underset{c^{\prime}, \boldsymbol{b}^{\prime}, \boldsymbol{g}^{\prime}}{\operatorname{argmin}}\left(\sum_{\boldsymbol{Q}}\left[I_{\boldsymbol{Q}, T}^{\mathrm{calc}}\left(c^{\prime}, \boldsymbol{b}^{\prime}, \boldsymbol{g}^{\prime}\right)-I_{\boldsymbol{Q}, T}^{\mathrm{expt}}\right]^{2}\right),
$$

where $I_{Q, T}^{\text {calc }}$ is the calculated intensity containing contributions from one-phonon and eventually $(n>1)$-phonon processes, and $c, \boldsymbol{b}$, and $\boldsymbol{g}$ are the fit parameters. In order to reduce the free parameters $c$ is constrained to the crystal symmetry, and $\boldsymbol{b}$ and $\boldsymbol{g}$ are kept constant in the vicinity of individual Bragg reflections. Such an approximation for $\boldsymbol{b}$ is justified by the fact that diffuse scattering due to additional contributions varies much less across reciprocal space than TDS for small $q$. The variation of corrections for absorption, polarization, and planar projection is small for the employed scattering geometry at small $q$ and thus justifies the approximation for $g$.

Solving the minimization problem Eq. (5) requires that the diffuse scattering is due to phonon scattering only. It can provide absolute values of the elastic constants if absolute intensities are known. If not, the elastic tensor is determined upon a single scaling factor and the absolute values of the elastic constants can be obtained if constrained to the adiabatic bulk modulus via the Reuss-VoigtHill relation [21].

Another option consists of measuring scattering intensities at slightly different temperatures. In fact, diffuse scattering from static disorder, air scattering, and fluorescence displays a much smaller temperature dependence than TDS, and can therefore be isolated in a difference measurement.

For experimental data at two slightly different temperatures $T_{1}$ and $T_{2}$ measured in the same geometry, we can solve the minimization problem,

$$
\begin{aligned}
c, \boldsymbol{b}_{2}, \boldsymbol{b}_{1}, \boldsymbol{g}= & \underset{c^{\prime}, \boldsymbol{b}_{2}^{\prime}, \boldsymbol{b}_{1}^{\prime}, \boldsymbol{g}^{\prime}}{\operatorname{argmin}}\left(\sum _ { \boldsymbol { Q } } \left\{\left[I_{\boldsymbol{Q}, T_{2}}^{\mathrm{calc}}\left(c^{\prime}, \boldsymbol{b}_{2}^{\prime}, \boldsymbol{g}^{\prime}\right)\right.\right.\right. \\
& \left.\left.\left.-I_{\boldsymbol{Q}, T_{1}}^{\mathrm{calc}}\left(c^{\prime}, \boldsymbol{b}_{1}^{\prime}, \boldsymbol{g}^{\prime}\right)\right]-\left(I_{\boldsymbol{Q}, T_{2}}^{\mathrm{expt}}-I_{\boldsymbol{Q}, T_{1}}^{\mathrm{expt}}\right)\right\}^{2}\right),
\end{aligned}
$$

which we call the multitemperature method. It neglects the variation of the elastic constants over the temperature interval $T_{1}, T_{2}$. The intensity difference must be compared to the variations of the Bose factor $\operatorname{coth}\left(\hbar \omega_{q, \nu} / 2 k T\right)$, which is approximately proportional to the temperature at high temperatures $(\hbar \omega>k T)$. This implies that, at constant $c$, the intensities measured at $T_{1}$ and $T_{2}$ are almost proportional to each other if one-phonon processes are predominant. Therefore, at such high temperatures, only ratios of elastic constants can be obtained if the scattering intensities are unknown on an absolute scale. At lower temperatures, 
the acoustic branches span two regions, a low-frequency one with $\hbar \omega \ll k T$ and one with higher frequencies $\hbar \omega>k T$. In such a case the intensities of the two measurements become linearly independent. The knowledge of $T$ determines the absolute scale to which $\omega$ compares, and, as a consequence, absolute values of the elastic constants can be obtained even for unknown absolute intensities. The temperature must be low enough so that the condition $\hbar \omega>k T$ is realized in regions of $\boldsymbol{q}$ where the elastic approximation is fulfilled.

Lattice dynamics calculations were carried out from first principles employing density functional perturbation theory [22] as implemented in the CASTEP code [23,24]. For both calcite and magnesium oxide, we used the local density approximation within a plane wave basis set and pseudopotentials of the optimized form [25]. The sampling of the electronic structure and the plane wave cutoff energy were chosen to ensure the convergence of internal forces to $<1 e^{-3} \mathrm{eV} / \AA$. Eigenfrequencies and eigenvectors of the acoustic branches close to $\Gamma$ were replaced by values obtained by the elastic approximation to ensure translational invariance. Fourier interpolation was employed for the computation of dynamical matrices at arbitrary $\boldsymbol{q}$. The calculated dynamical matrices were used to compute the Debye-Waller factors, and scattering intensities were calculated via Eqs. (1) and (2). The calculations are used for the correct selection of $\boldsymbol{q}$ values to be fitted and for the evaluation of multiple phonon scattering and contribution of optical phonons.

The determination of the elastic constants from TDS measured at a single temperature is demonstrated for a small calcite single crystal with trigonal symmetry (space group $R \overline{3} 2 / c$ ). For the fit, we consider the diffuse scattering intensity in the proximity of the 48 most intense Bragg reflections. The regions of interest (ROI) were parametrized by the absolute value of the reduced wave vector $\boldsymbol{q}$ in Cartesian coordinates and reduced units with $q_{i}=\Delta Q_{i} /\left|\boldsymbol{a}^{*}\right|$ and the data points selected according to the criteria $q \in\left[q_{\min }, q_{\max }\right]$. Here, $\boldsymbol{a}^{*}$ is the reciprocal lattice vector of the crystallographic axis $a$. The regions below $q_{\min }$ are excluded to minimize the contribution from elastic scattering while the regions above $q_{\max }$ are excluded in order to ensure the validity of the elastic approximation.

Experimental scattering intensities and calculated TDS from the fitted elastic constants in the elastic approximation for first order scattering are shown for selected regions of reciprocal space in Fig. 1. The plotted values are obtained from the irregular experimental data grid by inverting the interpolation equation. We do this using a few iterative steps and a Thikonov regularization term. Diffraction patterns that contain Bragg peaks were removed from the data treatment, because such images are affected by secondary scattering effects [26].

The elastic constants as obtained from fitting intensities of an ensemble of individual pixels are reported in Table I. Equation (5) was used to determine $c$ upon a single scaling
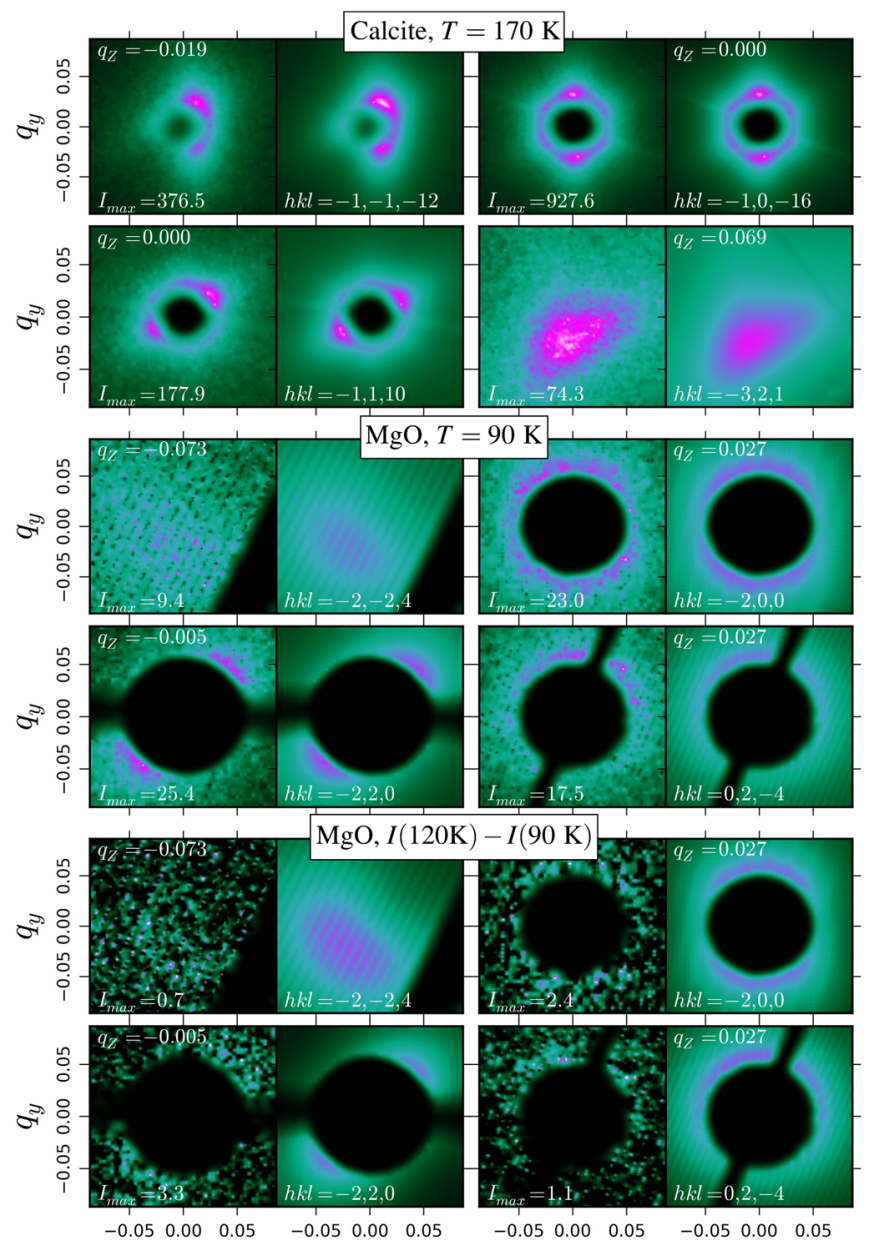

$q_{x}$

$q_{x}$

$q_{x}$

$q_{x}$

FIG. 1. Graphical rendering of measured diffuse scattering and calculated TDS. Considered cases are calcite at $T=170 \mathrm{~K}$ for a ROI of $q \in[0.03,0.15]$ (top panels), $\mathrm{MgO}$ with $q \in[0.06,0.15]$ at $T=90 \mathrm{~K}$ (middle panels), and with the multitemperature method at $T=90,120 \mathrm{~K}$ (bottom panels). Each image shows a cross section of the reciprocal space, in the neighborhood of the selected Bragg peak, and for a given $q_{z}=\Delta Q_{z} /\left|\boldsymbol{a}^{*}\right|$. The data are grouped by pairs, with the experimental and calculated intensity distribution on the left-hand and right-hand side, respectively. The scattering intensity is shown on a linear color scale from black (zero) to white (given maximal intensity $I_{\max }$ ). The reverse interpolation, which is only used for graphical rendering, results in some artifacts; for example, some intensity remains outside the ROI. The stripes visible at $h k l=\overline{2}, \overline{2}, 4$ and $0, \overline{2}, 4$ are due to the finite angular steps in the measurement, and each stripe corresponds to the contribution of a single diffraction pattern.

factor, and the absolute values were obtained by normalization to the known adiabatic bulk modulus $K$. Remarkable agreement with literature values determined from ultrasound measurements [27] are obtained for a ROI $q \in[0.06,0.15]$, which corresponds to approximately $2.1 \times 10^{7}$ intensity points. The difference is in the order of $1 \%$ for all elastic constants with exception of $c_{44}$. The higher limit $q_{\max }$ of the ROI is verified by $a b$ initio calculations. We therefore compute the scattering intensities from the calculated 
TABLE I. Elastic constants of calcite at $170 \mathrm{~K}$ in GPa. The values derived from fitting TDS are obtained for $q \in[0.06,0.15]$ and normalized by the adiabatic bulk modulus. Experimental reference values from ultrasound measurements (Ref. [27]) and the relative difference between the values derived from TDS and ultrasound are reported for comparison. Theoretical values were derived from fitting calculated TDS intensities within the same ROI ( $q \in[0.06,0.15])$ to the contribution of (i) acoustic branches only, (ii) all phonon branches, and (iii) acoustic branches including two-phonon scattering.

\begin{tabular}{lcccccc}
\hline \hline & TDS & $\begin{array}{c}\text { Reference } \\
{[27]}\end{array}$ & $\begin{array}{c}\text { Relative } \\
\text { difference }\end{array}$ & $\begin{array}{c}\text { Calculated } \\
\text { (i) }\end{array}$ & $\begin{array}{c}\text { Calculated } \\
\text { (ii) }\end{array}$ & $\begin{array}{c}\text { Calculated } \\
\text { (iii) }\end{array}$ \\
\hline$c_{11}$ & 156 & 155.1 & $0.6 \%$ & 154.3 & 153.5 & 154.2 \\
$c_{13}$ & 57.0 & 57.69 & $1.1 \%$ & 57.74 & 57.86 & 57.81 \\
$c_{15}$ & 21.2 & 21.51 & $1.6 \%$ & 21.11 & 20.86 & 20.94 \\
$c_{33}$ & 87.6 & 87.12 & $0.6 \%$ & 86.91 & 86.88 & 86.80 \\
$c_{44}$ & 35.7 & 34.42 & $3.7 \%$ & 33.95 & 33.60 & 33.75 \\
$c_{66}$ & 48.0 & 47.80 & $0.4 \%$ & 46.53 & 46.06 & 46.36 \\
\hline \hline
\end{tabular}

dynamical matrices and fit $c$ to it for different choices of ROI and compare the result to the expected value for $\lim q \rightarrow 0$. The lower limit $q_{\min }$ of the ROI must be chosen carefully, because very small momentum transfers might be affected by elastic scattering, as discussed further below. The contribution of optical phonons and multiple phonon scattering is evaluated by computing $I_{1}(\boldsymbol{Q})$ and $I_{2}(\boldsymbol{Q})$ including all phonon branches. A fit of elastic constants to these computed scattering intensities for the same ROI results in a maximal relative deviation of $1.2 \%$ if optical phonons are considered and only $0.8 \%$ maximal relative deviation if second order phonon scattering is included (see Table I).

The diffuse scattering in $\mathrm{MgO}$ is much less structured compared to calcite due to the higher cubic crystal symmetry (space group $F 4 / m \overline{3} 2 / m$ ); see Fig. 1. For fitting the elastic constants both methods, Eqs. (5) and (6), were employed considering diffuse scattering in the proximity of 78 of the most intense Bragg reflections. The results are reported in Table II. Fitting a single temperature with Eq. (5) is performed to obtain $c$ upon a uniform scaling factor at $T=90 \mathrm{~K}$. Because of the large discrepancy in $c_{12}$, the values are scaled to the experimental value of $c_{11}$ from ultrasound measurements instead of applying a scaling to the bulk modulus. The results are rather unsatisfactory compared to literature data [28]. This demonstrates the influence of additional diffuse scattering due to elastic scattering. We thus employ the multitemperature method [Eq. (6)] and fit the intensity differences of diffuse scattering measured at two close temperatures, 90 and $120 \mathrm{~K}$; see Fig. 1. The data seem noisy, but fitting the ensemble of approximately $1.7 \times 10^{7}$ pixels is sufficient for a well-converged result. Using this strategy, we obtain accurate values of the full elasticity tensor in absolute units, presented in Table II.

The results on calcite and magnesium oxide show that the full elasticity tensor can be measured with high accuracy by a rigorous data treatment of diffuse scattering in the proximity of Bragg reflections. The availability of single photon counting detectors with no readout noise together with a
TABLE II. Elastic constants of $\mathrm{MgO}$ in GPa measured at $T_{1}=$ $90 \mathrm{~K}$ and $T_{2}=120 \mathrm{~K}$. The elastic constants were fitted for $q \in$ $[0.06,0.15]$ applying the multitemperature method for the two temperatures $\left[I\left(T_{2}-T_{1}\right)\right]$ and for $T_{1}$ only [Fit $I\left(T_{1}\right)$, values rescaled to the reference value of $c_{11}$ ]. Ultrasound data [28] for the two temperatures and the relative difference between the average reference values and the results of the multitemperature method are listed for comparison.

\begin{tabular}{cccccc}
\hline \hline & & & Reference & Reference & \\
& Fit & Fit $I\left(T_{1}\right)$ & {$[28]$} & {$[28]$} & \\
\cline { 2 - 6 } & & $c_{11}$ & & & Relative \\
& $I\left(T_{2}\right)-I\left(T_{1}\right)$ & rescaled & $T=90 \mathrm{~K}$ & $T=120 \mathrm{~K}$ & difference \\
\hline$c_{11}$ & 300 & 306 & 306.1 & 305.4 & $2.0 \%$ \\
$c_{44}$ & 151 & 194 & 157.2 & 156.9 & $3.7 \%$ \\
$c_{12}$ & 89 & 39 & 94.07 & 94.26 & $5.7 \%$ \\
\hline \hline
\end{tabular}

large number of independent intensity points are crucial for successful experiments. For single crystals of very high quality, such as our investigated calcite crystal, the measurement of TDS at a single temperature can be sufficient to obtain the correct ratio of all elastic constants. The absolute values may then be obtained by a normalization to the adiabatic bulk modulus. The optimized choice of the ROI to be fitted is very important to ensure the validity of the elastic approximation and allows minimizing the contribution of elastic scattering. The best ROI can be found by successive adjustments of $q_{\min }$ and $q_{\max }$ and is verified here with the help of lattice dynamics calculations. Scattering contributions that vary slowly in reciprocal space can be taken into account in good approximation by a constant background to the diffuse scattering in the vicinity of individual Bragg reflections. Such contributions may include air scattering, fluorescence, and Compton scattering. This includes as well the contribution of optical phonons and higher order phonon scattering as shown by evaluating calculated dynamical matrices. Deconvolution might be applied for a more precise treatment of air scattering and beam shape that will allow for extending the ROI to smaller $q$ values, but this generally increases the noise level of the data. Secondary scattering effects like Bragg-diffuse scattering, where the Bragg reflected beam acts as a source of secondary diffuse scattering, are more difficult to treat and its contribution and scattering conditions are discussed elsewhere [26]. Here, we exclude all diffraction patterns that may be affected by such effects. If the diffuse scattering close to Bragg reflections is affected by elastic scattering, the multitemperature method might be a good choice. This method is based on the fact that TDS has a much stronger temperature dependence than other sources of diffuse scattering. Elastic and quasielastic contributions can thus be subtracted to a good approximation. Absolute values of the elasticity tensor can finally be obtained if the temperature interval is chosen such that the intensities become linear independent. This is shown for MgO. In addition to TDS, we observe elastic diffuse scattering, which we attribute to an imperfect crystal surface and defects. A careful measurement of absolute 
intensities may be a promising alternative to extract absolute values of the elastic constants but is likely less practical. In this study we compute the Debye-Waller factors from first principles. However, they can also be obtained from experiment using x-ray diffraction employing the same scattering geometry.

In summary, we have shown that accurate values of the full elasticity tensor can be obtained from a simple diffraction experiment on single crystals. Our method opens the perspective to determine elastic properties together with crystal structure investigations and thus under the same experimental conditions. This implies a broad applicability in material science, geophysics, and in the investigation of sound wave anomalies due to fundamental interactions in condensed matter physics. The achieved accuracy can compare with the standard methods such as ultrasound measurements and Brillouin scattering with the advantage of applicability to very small and opaque crystals of arbitrary shape. The proposed methodology can be extended to measurements at extreme conditions such as high pressures, high or low temperatures, or high magnetic fields. The contribution from the sample environment might be treated with the multitemperature method together with a deconvolution scheme for the treatment of temperature independent contributions. The application to high pressures is particularly interesting for the establishment of absolute pressure scales in a single experiment. Temperature dependent studies are possible within both methods. In the case of the multitemperature method, the temperature interval between the two measurements can be made very small by increasing statistics and momentum resolution. Extending the multitemperature method to three or more temperatures also allows for fitting the derivative of the elastic constants with respect to temperature. At very low temperatures the proposed strategy is expected to work well and is potentially very useful in the study of spin-lattice coupling. At temperatures relevant for geophysical processes, the scattering intensities will not be linear independent, but absolute values can be obtained if the adiabatic bulk modulus is known.

We thank Dmitry Chernyshov, Daniele de Sanctis, and Harald Reichert for fruitful discussions on measurement strategy and data treatment of diffuse scattering. Gael Goret is acknowledged for help in programing the first versions of the reciprocal space reconstruction routines. This work was supported by the European Community's Seventh Framework Programme (FP7/2007-2013) under Grant Agreement No. 290605 (PSI-FELLOW/COFUND) and resources of the European Synchrotron Radiation Facility.

*bjorn.wehinger@unige.ch

†mirone@esrf.fr

[1] F. I. Fedorov, Theory of Elastic Waves in Crystals (Plenum Press, New York, 1968).
[2] R. A. Cowley, Acoustic phonon instabilities and structural phase transitions, Phys. Rev. B 13, 4877 (1976).

[3] F. Birch, Elasticity and constitution of the Earth's interior, J. Geophys. Res. 57, 227 (1952).

[4] D. I. Bolef and J. de Klerk, Anomalies in the elastic constants and thermal expansion of chromium single crystals, Phys. Rev. 129, 1063 (1963).

[5] H. E. Bömmel, Ultrasonic attenuation in superconducting and normal-conducting tin at low temperatures, Phys. Rev. 100, 758 (1955).

[6] A. Migliori, W. M. Visscher, S. Wong, S. E. Brown, I. Tanaka, H. Kojima, and P. B. Allen, Complete Elastic Constants and Giant Softening of $c_{66}$ in Superconducting $\mathrm{La}_{1.86} \mathrm{Sr}_{0.14} \mathrm{CuO}_{4}$, Phys. Rev. Lett. 64, 2458 (1990).

[7] D. Almond and J. Rayne, Ultrasonic attenuation in linear antiferromagnet $\mathrm{CsNiCl}_{3}$, Phys. Lett. A 54, 295 (1975).

[8] B. Wolf, S. Zherlitsyn, S. Schmidt, and B. Lüthi, Soundwave propagation in pulsed magnetic fields in $\mathrm{CsCuCl}_{3}$, Europhys. Lett. 48, 182 (1999).

[9] B. Wehinger, A. Bosak, S. Nazzareni, D. Antonangeli, A. Mirone, S. L. Chaplot, R. Mittal, E. Ohtani, A. Shatskiy, S. Saxena, S. Ghose, and M. Krisch, Dynamical and elastic properties of $\mathrm{MgSiO}_{3}$ perovskite (bridgmanite), Geophys. Res. Lett. 43, 2568 (2016).

[10] D. Antonangeli, M. Krisch, G. Fiquet, D. L. Farber, C. M. Aracne, J. Badro, F. Occelli, and H. Requardt, Elasticity of Cobalt at High Pressure Studied by Inelastic X-Ray Scattering, Phys. Rev. Lett. 93, 215505 (2004).

[11] D. Antonangeli, T. Komabayashi, F. Occelli, E. Borissenko, A. C. Walters, G. Fiquet, and Y. Fei, Simultaneous sound velocity and density measurements of hcp iron up to $93 \mathrm{GPa}$ and $1100 \mathrm{~K}$ : An experimental test of the Birch's law at high temperature, Earth Planet. Sci. Lett. 331-332, 210 (2012).

[12] W. A. Wooster, Diffuse X-Ray Reflections from Crystals (Oxford University Press, New York, 1962).

[13] H. Faxén, Die bei Interferenz von Röntgenstrahlen infolge der Wärmebewegung entstehende Streustrahlung, Z. Phys. 17, 266 (1923).

[14] I. Waller, Zur Frage der Einwirkung der Wärmebewegung auf die Interferenz von Röntgenstrahlen, Z. Phys. 17, 398 (1923).

[15] P. Olmer, Dispersion des vitesses des ondes acoustiques dans l'aluminium, Acta Crystallogr. 1, 57 (1948).

[16] M. Holt, Z. Wu, H. Hong, P. Zschack, P. Jemian, J. Tischler, H. Chen, and T.C. Chiang, Determination of Phonon Dispersions from X-Ray Transmission Scattering: The Example of Silicon, Phys. Rev. Lett. 83, 3317 (1999).

[17] R. Q. Xu and T.C. Chiang, Determination of phonon dispersion relations by $\mathrm{X}$-ray thermal diffuse scattering, Z. Kristallogr. 220, 1009 (2005).

[18] A. Bosak, D. Chernyshov, B. Wehinger, B. Winkler, M. L. Tacon, and M. Krisch, In-between Bragg reflections: Thermal diffuse scattering and vibrational spectroscopy with x-rays, J. Phys. D 48, 504003 (2015).

[19] A. Bosak and D. Chernyshov, On model-free reconstruction of lattice dynamics from thermal diffuse scattering, Acta Crystallogr. Sect. A 64, 598 (2008).

[20] D. de Sanctis, A. Beteva, H. Caserotto, F. Dobias, J. Gabadinho, T. Giraud, A. Gobbo, M. Guijarro, M. Lentini, B. Lavault, T. Mairs, S. McSweeney, S. Petitdemange, 
V. Rey-Bakaikoa, J. Surr, P. Theveneau, G. Leonard, and C. Mueller-Dieckmann, ID29: A high-intensity highly automated ESRF beamline for macromolecular crystallography experiments exploiting anomalous scattering, J. Synchrotron Radiat. 19, 455 (2012).

[21] R. Hill, The elastic behaviour of a crystalline aggregate, Proc. Phys. Soc. London Sect. A 65, 349 (1952).

[22] X. Gonze and C. Lee, Dynamical matrices, Born effective charges, dielectric permittivity tensors, and interatomic force constants from density-functional perturbation theory, Phys. Rev. B 55, 10355 (1997).

[23] S. Clark, M. Segall, C. Pickard, P. Hasnip, M. Probert, K. Refson, and M. Payne, First principles methods using CASTEP, Z. Kristallogr. 220, 567 (2005).
[24] K. Refson, P. R. Tulip, and S. J. Clark, Variational densityfunctional perturbation theory for dielectrics and lattice dynamics, Phys. Rev. B 73, 155114 (2006).

[25] A. M. Rappe, K. M. Rabe, E. Kaxiras, and J. D. Joannopoulos, Optimized pseudopotentials, Phys. Rev. B 41, 1227 (1990).

[26] I. B. Ramsteiner, A. Schöps, H. Reichert, H. Dosch, V. Honkimäki, Z. Zhong, and J. B. Hastings, High-energy X-ray diffuse scattering, J. Appl. Crystallogr. 42, 392 (2009).

[27] D. P. Dandekar and A. L. Ruoff, Temperature dependence of the elastic constants of calcite between $160^{\circ}$ and $300^{\circ} \mathrm{K}$, J. Appl. Phys. 39, 6004 (1968).

[28] K. Marklund and S. A. Mahmoud, Elastic constants of magnesium oxide, Phys. Scr. 3, 75 (1971). 